\title{
Indisputable Proof about the Effects of Eye Rubbing on the Cornea, the Lens and the Globe
}

\author{
Edna Joyce Santos* \\ Department of Ophthalmology Union Medical and Dental Center Ras Al Khaimah, United Arab Emirates
}

*Corresponding author: Edna Joyce Santos, Department of Ophthalmology Union Medical and Dental Center Ras Al Khaimah, United Arab Emirates.

To Cite This Article: Edna Joyce Santos, Indisputable Proof about the Effects of Eye Rubbing on the Cornea, the Lens and the Globe. Am J Biomed Sci \& Res. 2020 - 7(1). AJBSR.MS.ID.001120. DOI: 10.34297/AJBSR.2020.07.001120.

Received: 眥 January 01, 2020; Published: 战 January 29, 2020

\section{Introduction}

As we herald the beginning of the new decade, we welcome a lot of innovations in the field of ophthalmology, especially the dawn of digitalization in the healthcare industry. And it is through one of these dynamic technologies, Magnetic Resonance Imaging, which saw prominence during the last two decades, that the myth about eye rubbing not influencing the development of keratoconus has been completely demystified and debunked. Dr. Damien Gatinel, Dr. Giullaume Debellumaniere and Dr. Julian Savatovsky of the Rothschild Foundation Hospital in France showed in You Tube in November 10, 2019 the actual footage of a healthy volunteer rubbing his eye while explicit digital images were being taken and recorded using MRI https://youtu.be/tM4z3MYZeNK.

The images were very impressive and revealed how the cornea, the lens, the globe and surrounding orbital structures were distorted by the seemingly innocuous gesture of rubbing the eyes. Eye rubbing is usually an attempt to get a slight sense of relief for the unpleasant condition afflicting the individual's eye/eyes at that moment. Keratoconus is a thinning and deforming of the cornea. The importance of eye rubbing in the development of keratoconus has been underestimated, if not completely ignored by the medical community. Now, through the striking images captured in real time, there is indisputable proof that keratoconus is, without a doubt, a disease of friction. The propensity for individuals to develop the disease, I believe, depends on the congenital structure of the cornea the individual is born with, which answers the question on why some persons are prone to develop and progress into keratoconus while others don't.

There are several types of eye rubbing:

Type $1 \quad$ : Rubbing the eye with one finger

Type 2 : Knuckle rub

Type 3 : Palm rub
Type $4 \quad$ : Back of the hand rub

Type 5 : Shoulder rub

Type $6 \quad$ : Face in the pillow rub

Several causes for chronic eye rubbing have been raised, namely, atopy/allergy, dry eye, contact lens wear, pollution, presence of foreign body, night compression, habit/compulsory and pleasant sensation, which leads to bio-cellular and bio-mechanical consequences.

a) Bio-cellular consequences (extracellular matrix degradation, impaired surface barrier, collagenase and enzymatic activity, cell mechanosensitivity, reduction of proteoglycans and microfibrils, and reduction of collagen maintenance)

b) Bio-mechanical consequences (shearing forces lead to viscoelastic and ground substance displacement, fiber and fibril slippage, transient deformation, corneal weakening reduction of collagen maintenance and central stromal thinning)

Both ultimately and unfortunately lead to corneal macroscopic deformity like keratoconus. In some instances, there is a disparity in the corneal distortion in both eyes, and the more affected eye would be the one the patient sleeps on.

\section{Conclusion}

So, this is a timely reminder to our colleagues to admonish their patients on the dangers of eye rubbing, unless they want to rub their eyes blind. It is, therefore, recommended that we educate our patients to be more vigilant in order to preserve their corneal integrity, prevent eye asymmetry and conserve their vision. 